\title{
Prevalencia de lesiones maxilares en ortopantomografía de pacien- tes que acuden a las clínicas odontológicas de la UNAH y HEU, 2016-2017
}

Prevalence of maxillary lesions in orthopantomography of patients attending dental clinics of UNAH and HEU, 2016-2017

DOI 10.5377pc.v0i16.8098

Roberto Carlos Escobar Díaz ${ }^{1}$

Hugo Humberto Romero ${ }^{2}$

Ricardo Antonio Aguilar ${ }^{3}$

\section{RESUMEN}

Las lesiones maxilofaciales son un grupo heterogéneo de alteraciones que son observables en las ortopantomografías como radiolucidez, radiopacidad o una combinación de ambos. Determinar la prevalencia de lesiones maxilares de pacientes que acudieron a las clínicas odontológicas de la UNAH y HEU durante 2016-2017. Se realizó un estudio con enfoque cuantitativo, alcance descriptivo, diseño transversal observacional y retrospectivo de radiografías panorámicas digitales, explorando alteraciones maxilofaciales. Se realizó la caracterización de su presentación según sexo y edad. Se analizaron 326 ortopantomografías digitales, 111 (34\%) de hombres y $215(65.9 \%)$ de mujeres. Edades límite de 5 y 80 años. Se encontró que $86(26.3 \%)$ presentaban lesiones en los maxilares, 37 (43\%) en hombres y $49(56.9 \%)$ en mujeres. El grupo etario de 21 a 30 años fue el que más lesiones presentó 21 (24.4\%), De acuerdo a las instituciones 229 personas eran de la UNAH con $24.4 \%$ lesiones, y 97 del HEU con 30.92\% lesiones maxilares. El tipo de lesión más común fue el radiolúcido con un $65 \%$. Las lesiones en el complejo maxilofacial presentan una variedad de patrones radiográficos.

Palabras clave: lesiones maxilares, ortopantomografía, radiolucidez, radiopacidad.

\footnotetext{
${ }^{1}$ Autor, beneficiario beca estudiante de pregrado de la DICYP, UNAH roberto.escobar@unah.hn

2 Asesor teórico. Cirujano Maxilofacial, UNAH cmfalvarenga@gmail.com

${ }^{3}$ Asesor teórico. Cirujano y Patólogo Oral, UNAH implantsky@hotmail.com
} 


\section{ABSTRACT}

Maxillofacial lesions are a heterogeneous group of alterations that are observable in orthopantomographies such as radiolucency, radiopacity or a combination of both. To determine the prevalence of maxillary lesions of patients who attended the dental clinics of the UNAH and HEU during 2016-2017. A study was carried out with a quantitative approach, descriptive scope, cross-sectional observational and retrospective design of digital panoramic radiographs, exploring maxillofacial alterations. The characterization of its presentation was carried out according to sex and age. 326 digital orthopantomographies were analyzed, $111(34 \%)$ of men and $215(65.9 \%)$ of women. Limit ages of 5 and 80 years. It was found that $86(26.3 \%)$ had lesions in the jaws, $37(43 \%)$ in men and $49(56.9 \%)$ in women. The age group from 21 to 30 years was the one that presented more injuries 21 (24.4\%), According to the institutions 229 people were from the UNAH with $24.4 \%$ injuries, and 97 from the HEU with $30.92 \%$ maxillary lesions. The most common type of injury was radiolucent with $65 \%$. Lesions in the maxillofacial complex present a variety of radiographic patterns.

Keywords: maxillary lesions, orthopantomography, radiolucency, radiopacity. 


\section{INTRODUCCIÓN}

Las lesiones maxilofaciales son un grupo heterogéneo de alteraciones que afectan los tejidos duros y blandos de la cara que van desde lesiones quísticas hasta tumorales y pueden estar presentes en todo tipo de personas, incluyendo niños, adultos y ancianos, muchas de estas alteraciones son llamados silenciosas ya que se inicia y evoluciona sin que la persona tenga conocimiento, porque no provoca síntomas (Sinche A, 2014) y pueden comprometer el crecimiento y desarrollo de los maxilares. Estos hallazgos pueden llegar a constituir un problema de salud en los pacientes. (Ponce de León Pérez, María Alejandra, 2014) Además, existen muchas lesiones que en sus estadios iniciales puede aparentar ser un quiste, sin embargo, no es hasta que la lesión ha crecido cuando se suele diagnosticar un tumor.

El uso de radiografías en la práctica odontológica como complemento a la descripción de signos y síntomas durante la exploración física de los pacientes, se constituye en un elemento de gran utilidad, necesario para el establecimiento de un adecuado diagnóstico. Las radiografías utilizadas en odontología se clasifican en intraorales y extraorales, (Jiménez Ortiz, José L. Herrera Silva, Jorge C. et al, 2017) La Ortopantomografía o Radiografía panorámica (RP) es la proyección radiográfica extraoral que se utiliza con mayor frecuencia en odontología, es una técnica radiológica que nos permite tener una visión general de las estructuras maxilares y sus relaciones dentales. Brinda una amplia visión de las estructuras de la cara media e inferior y su relación con el complejo maxilofacial (Sánchez Trocino, Benjamín. Hernández, Javier. et al, 2013). Constituye el "Gold Standard" para la evaluación inicial llevada a cabo en odontología porque permite la observación del tejido óseo y dental normal, y anormal de los maxilares. (Zúñiga, 2017)

Las lesiones del complejo óseo maxilofacial son observadas en la Ortopantomografía como radiolúcidas cuando debido a la estructura ósea el haz de rayos $\mathrm{x}$ pasa fácilmente, radiopacas cuando se observa un imagen blanca que no dejar pasar el haz de rayos x y mixtas cuando existe una combinación de ambos (Sinche A, 2014) Entre los hallazgos que se pueden encontrar en ortopantomografías están las imágenes compatibles con lesiones quísticas, tumorales y anomalías dentales. Los quistes odontogénicos son cavidades patológicas recubiertas por epitelio, originados a partir de componentes epiteliales del aparato odontogénico o de restos celulares que quedan atrapados en el proceso de fusión dentro del hueso o en los tejidos gingivales periféricos. Constituyen una de las principales causas de destrucción de los huesos maxilares, y se manifiestan como una radiolucidez con bordes definidos (Peters, F I. 
et al, 2012) La prevalencia de los quistes odontogénicos varía según distribución geográfica, género y edad, entre otros.

Dentro de los hallazgos radiográficos tumorales destaca el ameloblastoma, que en fases iníciales está perfectamente delimitado y suele estar rodeado por un borde hiperestésico, lo que indica que su crecimiento es lento. Radiográficamente, el ameloblastoma ofrece una imagen radiolúcida, variable en su aspecto, la forma más típica es la multilocular, donde la apariencia es en pompas de jabón los límites de la imagen pueden ser lisos o pueden tener ciertas irregularidades, (Sinche A, 2014). La literatura nacional es escasa ya que no existe ninguna investigación que determine la frecuencia de lesiones maxilares en ortopantomografía, esto con el fin de dar bases epidemiológicas para orientar los servicios de atención en salud ya que permite la medición de la necesidad de cirugía maxilofacial, (Alvarado, Esteban. Chanis, Jonattan, 2016) Fomentar el uso de RP en la evaluación de rutina permite descartar patologías asintomáticas y reducir costos detectándolas en sus estados iniciales, y no cuando ya están sintomáticas y de gran tamaño. El propósito es determinar la prevalencia de lesiones maxilares en ortopantomografía de pacientes que acuden a las clínicas odontológicas de la facultad de odontología de la UNAH y el Hospital Escuela Universitario (HEU) durante 2016-2017.

\section{MATERIALES Y MÉTODOS}

Se realizó un estudio con enfoque cuantitativo, alcance descriptivo, diseño transversal observacional y retrospectivo. Se examinaron 326 radiografías que fueron aleatoriamente seleccionadas de una población de 4152 personas que asistieron a UNAH y HEU durante 2016 y 2017. El tamaño de la muestra se obtuvo con un error del 5\%, distribución de 50 y nivel de confianza 95\%. Los grupos etarios fueron clasificados por decenios, el rango fue de 5 a 80 años de edad. Las radiografías panorámicas (RP) fueron obtenidas en un centro radiológico de la ciudad de Tegucigalpa, Honduras. Para la selección de las RP se consideró que cada radiografía contenga la especificación de datos como sexo, edad, institución, tipo de lesión, ubicación, forma y número de lesión.

Los criterios de inclusión fueron: radiografías panorámicas digitales con adecuada densidad y contraste, de pacientes de ambos sexos, entre 5 y 80 años. Criterios de exclusión: RP de pacientes con traumas, con enfermedades que afecten a los maxilares, RP que posean poca definición imagenológica. Tres clínicos un estudiante y dos 
profesores realizaron la evaluación radiográfica, considerando sólo como anormalidades, aquellos casos en que existió una concordancia completa de la existencia de la lesión entre los tres observadores. Con un aparato de rayos $X$ sirona. La muestra de radiografías fue examinada en una Tablet Samsung 9.6 pulgadas con pantalla retina retroiluminada por LED y la información recolectada fue almacenada en una computadora Dell Inspiron 14 con pantalla True Life HD 14 pulgadas.

Los datos extraídos fueron almacenados en base de datos de Excel 2016 con las variables: sexo, edad, institución, tipo de lesión, ubicación anatómica, forma de la lesión y cantidad de lesiones por radiografía. El análisis estadístico se realizó con el programa SPSS versión 24, considerando la distribución de frecuencias absolutas y relativas de las variables estudiadas y determinación de medidas resumen con la media y de dispersión como la desviación estándar en aquellas variables que correspondiese. El protocolo de investigación fue evaluado y aprobado por el departamento de proyectos de investigación de la DICYP. El presente estudio no presentó implicaciones éticas, debido a que se mantuvo la confidencialidad de la información y se codificó las radiografías de los participantes del estudio.

\section{RESULTADOS}

Se analizaron 326 ortopantomografías digitales, 111 (34\%) de hombres y 215 (65.9\%) de mujeres. Las edades oscilan entre 5 años y 80 años, con una media de 33 años. De las 326 (100\%) Ortopantomografías, 86 (26.3\%) presentaron lesiones en los maxilares, de los cuales $37(43 \%)$ en hombres y 49 (56.9\%) en mujeres. El grupo etario que presentó el mayor número de personas fue de 21 a 30 años con 101 radiografías (30.9\%) y a la vez fue el que más lesiones presentó con $21(24.4 \%)$, le siguen en orden descendente el grupo de 11 a 20 con 17 (19.7\%) lesiones, y 51 a 60 con 16 (18.6\%) lesiones. Tabla 1.

En relación a las instituciones 229 (70.2\%) personas fueron de la UNAH, 74 (32\%) hombres y $155(50.2 \%)$ mujeres, se encontraron 56 lesiones, 22 (39\%) en hombres y 34 (60.7\%) en mujeres. El total de personas atendidas en HEU fue $97(29.7 \%)$ de los cuales $37(38.1 \%)$ en hombres y $60(61.8 \%)$ en mujeres, $30(30.92 \%)$ personas presentaron lesiones, $15(50 \%)$ en hombres y $15(50 \%)$ mujeres. Tabla 2 . De acuerdo al tipo de lesión encontrada en ambas instituciones $56(65 \%)$ eran radiolúcidas, 23 $(26.7 \%)$ radiopacas y $7(8.1 \%)$ presentaban un patrón mixto. Tabla 3 . Se observa radiografía de un paciente de 16 años con lesión mixta en maxilar superior. Figura 1. 
Tabla 1. Distribución según edad y sexo

\begin{tabular}{|c|c|c|c|c|c|c|}
\cline { 2 - 7 } \multicolumn{1}{c|}{} & \multicolumn{5}{c|}{ Sexo } \\
\hline $\begin{array}{c}\text { Grupos } \\
\text { de edad }\end{array}$ & $\begin{array}{c}\text { Hombre } \\
\mathbf{N}\end{array}$ & Mujer N & Total N (\%) & $\begin{array}{c}\text { Hombre } \\
\mathbf{n}\end{array}$ & Mujer n & Total n (\%) \\
\hline $\mathbf{5 - 1 0}$ & 6 & 8 & $14(4.2 \%)$ & 0 & 0 & $0(0 \%)$ \\
\hline $\mathbf{1 1 - 2 0}$ & 23 & 43 & $66(20.2 \%)$ & 6 & 11 & $17(19.7 \%)$ \\
\hline $\mathbf{2 1 - 3 0}$ & 32 & 69 & $101(30.9 \%)$ & 12 & 9 & $21(24.4 \%)$ \\
\hline $\mathbf{3 1 - 4 0}$ & 16 & 30 & $46(14.1 \%)$ & 9 & 5 & $14(16.2 \%)$ \\
\hline $\mathbf{4 1 - 5 0}$ & 10 & 26 & $36(11 \%)$ & 3 & 10 & $13(15.1 \%)$ \\
\hline $\mathbf{5 1 - 6 0}$ & 13 & 25 & $38(11.6 \%)$ & 5 & 11 & $16(18.6 \%)$ \\
\hline $\mathbf{6 1 - 7 0}$ & 8 & 9 & $17(5.2 \%)$ & 2 & 2 & $4(4.6 \%)$ \\
\hline $\mathbf{7 1 - 8 0}$ & 3 & 5 & $8(2.4 \%)$ & 0 & 1 & $1(1.1 \%)$ \\
\hline Total & 111 & 215 & $326(100 \%)$ & 37 & 49 & $86(100 \%)$ \\
& $(34 \%)$ & $(65.9 \%)$ & & $(43 \%)$ & $(56.9 \%)$ & \\
\hline
\end{tabular}

$\mathrm{N}$ : Casos estudiados.

$\mathrm{n}$ : Casos con presencia de lesión maxilar.

Fuente: elaboración propia

Tabla 2. Distribución según Institución y sexo

\begin{tabular}{|c|c|c|c|c|c|c|}
\cline { 2 - 7 } \multicolumn{1}{c|}{} & \multicolumn{7}{c|}{ Sexo } \\
\hline Institución & Hombre N & Mujer N & Total N (\%) & Hombre $\mathbf{~}$ & Mujer $\mathbf{n}$ & Total $\mathbf{n}(\%)$ \\
\hline HEU & 37 & 60 & $97(29.7 \%)$ & 15 & 15 & $30(34.8 \%)$ \\
\hline UNAH & 74 & 155 & $229(70.2 \%)$ & 22 & 34 & $56(65.1 \%)$ \\
\hline Total & 111 & 215 & $\mathbf{3 2 6}(100 \%)$ & 37 & 49 & $\mathbf{8 6}(100 \%)$ \\
\hline
\end{tabular}

$\mathrm{N}$ : Casos estudiados.

$\mathrm{n}$ : Casos con presencia de lesión maxilar.

Fuente: elaboración propia

Por su localización 53 lesiones estaban presentes en el maxilar superior 23 (47.1\%) en la región anterior y 28 (52.8\%) en la región posterior. En la mandíbula se presentaron $6(19.4 \%)$ en la región anterior, 24 (69.4\%) en el cuerpo, 2 (5.5\%) en el ángulo, 2 $(5.5 \%)$ en la rama. De acuerdo a su forma radiográfica se encontró que $39(45.3 \%)$ presentaron un patrón radiográfico unilocular, 1 (1.16\%) fue multilocular y $45(52.3 \%)$ un patrón irregular. Tabla 4. 
Tabla 3. Distribución según tipo de lesión y sexo

\begin{tabular}{|c|c|c|c|}
\cline { 2 - 4 } \multicolumn{1}{c|}{} & \multicolumn{3}{c|}{ Sexo } \\
\hline Tipo de lesión & Hombre & Mujer & Total (\%) \\
\hline Radiolúcida & 24 & 32 & $56(65.1 \%)$ \\
\hline Radiopaca & 8 & 15 & $23(26.7 \%)$ \\
\hline Mixta & 5 & 2 & $7(8.1 \%)$ \\
\hline Total & 37 & 49 & $\mathbf{8 6 ( 1 0 0 \% )}$ \\
\hline
\end{tabular}

Fuente: elaboración propia

En relación con el número de lesiones presentes en las radiografías, 57 (66.2\%) presentaron una lesión, 17 (19.7\%) presentaban dos lesiones y 13 (15.1\%) presentaban más de 3 lesiones en ambos maxilares.

Figura 1. Ortopantomografía con presencia de lesión maxilar.

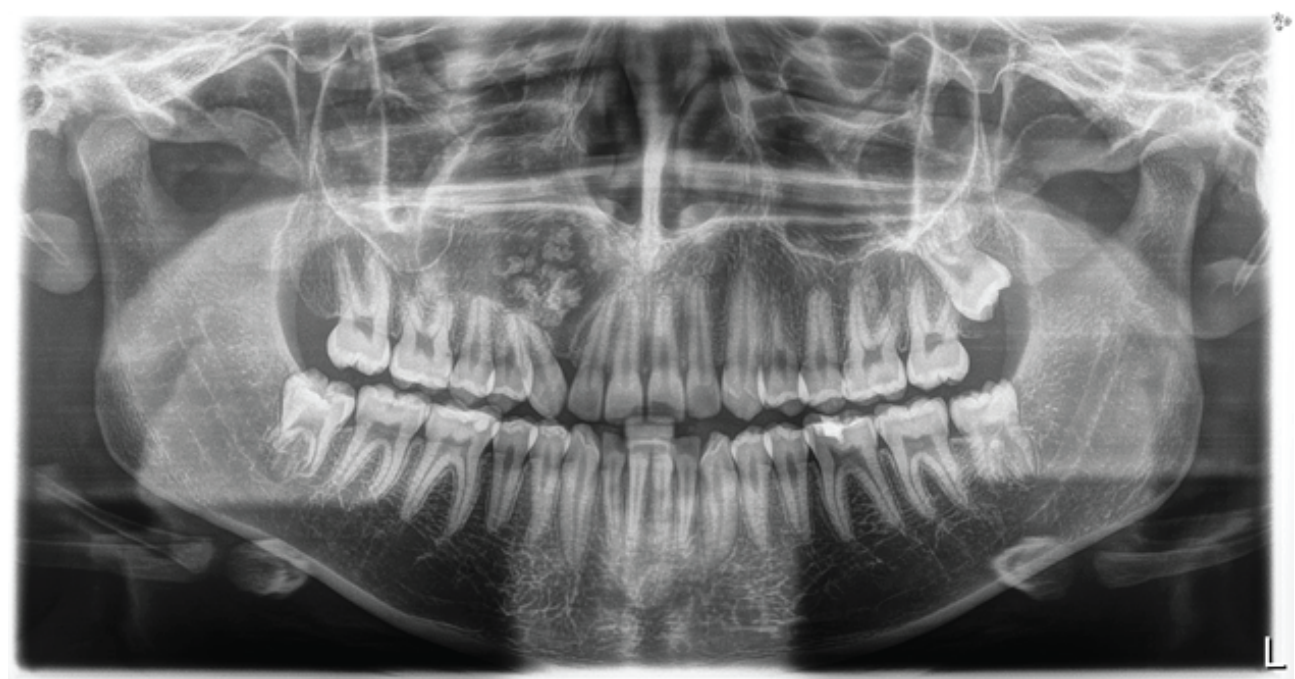

Se observa lesión tipo mixta, de forma irregular localizada en la región anterior del maxilar superior, en un paciente masculino de 16 años, HEU

Fuente: base de datos del estudio. 
Tabla 4. Distribución según tipo, forma y localización de la lesión

\begin{tabular}{|c|c|c|c|c|c|c|c|c|}
\hline \multirow[b]{2}{*}{ Tipo de lesión } & \multirow[b]{2}{*}{$\begin{array}{l}\text { Forma de } \\
\text { lesión }\end{array}$} & \multicolumn{6}{|c|}{ Localización de lesión } & \multirow[b]{2}{*}{ Tota } \\
\hline & & $\begin{array}{l}\text { Maxilar } \\
\text { Anterior }\end{array}$ & $\begin{array}{l}\text { Maxilar } \\
\text { Posterior }\end{array}$ & $\begin{array}{l}\text { Mandibular } \\
\text { Anterior }\end{array}$ & $\begin{array}{l}\text { Mandibular } \\
\text { Cuerpo }\end{array}$ & $\begin{array}{l}\text { Mandibular } \\
\text { Ángulo }\end{array}$ & $\begin{array}{l}\text { Mandibular } \\
\text { Rama }\end{array}$ & \\
\hline \multirow[t]{4}{*}{ Radiolúcida } & Unilocular & 4 & 5 & 3 & 5 & 0 & 1 & 18 \\
\hline & Multilocular & 0 & 1 & 0 & 0 & 0 & 0 & 1 \\
\hline & Irregular & 13 & 6 & 2 & 13 & 1 & 1 & 36 \\
\hline & Total & 17 & 12 & 5 & 18 & 1 & 2 & 56 \\
\hline \multirow[t]{4}{*}{ Radiopaca } & Unilocular & 3 & 10 & 1 & 3 & 1 & 0 & 18 \\
\hline & Multilocular & 0 & 0 & 0 & 0 & 0 & 0 & 0 \\
\hline & Irregular & 1 & 1 & 0 & 3 & 0 & 0 & 5 \\
\hline & Total & 4 & 11 & 1 & 6 & 1 & 0 & 23 \\
\hline \multirow[t]{4}{*}{ Mixta } & Unilocular & 1 & 2 & 0 & 0 & 0 & 0 & 3 \\
\hline & Multilocular & 0 & 0 & 0 & 0 & 0 & 0 & 0 \\
\hline & Irregular & 1 & 3 & 0 & 0 & 0 & 0 & 4 \\
\hline & Total & 2 & 5 & 0 & 0 & 0 & 0 & 7 \\
\hline \multirow[t]{4}{*}{ Total } & Unilocular & 8 & 17 & 4 & 8 & 1 & 1 & 39 \\
\hline & Multilocular & 0 & 1 & 0 & 0 & 0 & 0 & 1 \\
\hline & Irregular & 15 & 10 & 2 & 16 & 1 & 1 & 45 \\
\hline & Total & 23 & 28 & 6 & 24 & 2 & 2 & 86 \\
\hline
\end{tabular}

Fuente: elaboración propia

\section{Discusión}

Los estudios sobre la prevalencia de lesiones orales y maxilofaciales son importantes para caracterizar las lesiones más frecuentes en poblaciones específicas, son herramientas que permiten mejorar la comprensión de las enfermedades y establecer protocolos de tratamiento. (Amadeu, Josi K. Schussel, Juliana L. et al, 2015) En el presente estudio, se logró revisar la totalidad de radiografías del periodo estudiado, con lo cual se creó una base de datos que no existía en HEU y la UNAH, aportando información relevante a la estadística nacional en lo que a salud maxilofacial se refiere. Las limitantes del estudio son que no lleva un componente diagnóstico que permita determinar qué lesiones corresponden a patología específicas.

La radiografía panorámica es una técnica radiográfica que provee una visualización adecuada de las estructuras de la región maxilomandibular de manera completa. La RP es utilizada de manera rutinaria tanto a nivel privado como institucional, para llevar a cabo una evaluación de la dentición completa, el hueso alveolar, articulaciones temporomandibulares, y de las estructuras adyacentes de una manera fácil y rápida. (Jímenez Órtiz, José L. et al, 2017) Los hallazgos radiográficos que se pueden apreciar con mayor facilidad son aquellos que se encuentran en las estructuras óseas como son los senos maxilares y las fosas nasales, estos son fácilmente identificables en una placa panorámica(Sinche A, 2014) 
En un estudio realizado en Perú hubo mayor prevalencia de hallazgos en hombres con 576 casos (58.01\%), mientras que en mujeres hubo 417 casos (41.99\%), (Ponce de León Pérez, María Alejandra. 2014) en contraste con este estudio hubo mayor prevalencia en mujeres con $49(56.9 \%)$ y $37(43 \%)$ en hombres. En un estudio sobre lesiones maxilofaciales que se realizó a adolescentes en un periodo de 20 años se encontró que la tercera ubicación más común es el maxilar (Amadeu, Josi K. et al, 2015) en el presente estudio también la ubicación más común fue el maxilar en la región posterior con $28(32,5 \%)$ y la región anterior fue la tercera más común con 23 $(26.7 \%)$ hallazgos. En un estudio realizado en Chile sobre tumores odontogénicos, según zona anatómica de presentación de lesiones benignas, la más frecuente corresponde a la zona molar mandibular, (54,8\%) (Thiers, LC. Sotomayor, CC. Peters, FI. et al 2013) mientras que en el presente estudio la ubicación del cuerpo mandibular fue la segunda más común $24(27.9 \%)$.

En un estudio realizado en México se encontró una incidencia de 15 lesiones radiotransparentes $(2,90 \%)$ con una distribución según sexo de 4 en hombres $(26,66 \%)$ y 11 en mujeres $(73,33$. \%). En la mandibula, 43 lesiones fueron radiotransparentes $(8,33 \%)$ con una distribución por sexo de 18 en hombres $(41,86 \%)$ y 25 mujeres $(58,13 \%) .8$ lesiones radiopacas en el maxilar (1.55\%) de las cuales una ocurrió en hombres (12.5\%) y 7 en mujeres $(87.5 \%$ ) Con respecto a las lesiones radiopacas de la mandíbula, se encontró una incidencia de 49 lesiones (9.49\%) de las cuales 18 fueron en hombres (36.73\%) y 31 en mujeres (63.26\%) (Sanchez Trocino, Benjamin. et al, 2013) En comparación con el presente estudio en el que se encontró 56 (65\%) lesiones eran radiolúcidas, con una distribución por sexo de $24(42.8 \%)$ en hombres y $32(57.1 \%)$ en mujeres, $23(26.7 \%)$ lesiones radiopacas, $8(34.7 \%)$ en hombres y 15 $(65.2 \%)$ en mujeres y, $7(8.1 \%)$ lesiones presentaban un patrón mixto con $5(71.4 \%)$ en hombres y $2(28.5 \%)$ en mujeres.

Es necesario realizar futuras investigaciones en este ámbito, indagar en aspectos relacionados con causalidad y significancia estadística que puedan crear hipótesis en cuanto a la presentación de estas lesiones en la población y obtener patrones de distribución y asociación más exactos.

\section{CONCLUSIONES}

- De acuerdo a los resultados obtenidos en el estudio la prevalencia de lesiones en los maxilares representa más de un cuarto por ciento de la población estudiada. 
- Las lesiones maxilofaciales presentan distintos patrones radiográficos lo que dificulta su diagnóstico diferencial con múltiples patologías.

\section{AGRADECIMIENTOS.}

Ala Lic. Martha Edelea Díaz (Q.D.E.P.) por la orientación y apoyo en la realización del estudio, al Dr. Lino Carmenate por el tiempo brindado en la asesoría metodológica, y a DSD Radiodiagnóstico por permitir acceso a las bases de datos y poder recolectar las muestras para el estudio.

\section{REFERENCIAS BIBLIOGRÁFICAS}

Alvarado, Esteban. Chanis, Jonattan. Barrientos, Silvia. Rodríguez, Adriana. (2016): Hallazgos compatibles con material de osteosíntesis en 10.000 radiografías panorámicas: un estudio descriptivo en Bogotá, Colombia. En: Odontoestomatología 18 (27), pág. 34-41. Amadeu, Josi K. Schussel, Juliana L. Piazzetta, Cleto M. Torres-Pereira, Cassius C. Amenábar, José M. (2015): Lesiones Orales y del Complejo Maxilofacial en Adolescentes: Un Estudio Retrospectivo de 20 Años. En: Int. J. Odontostomat. 9 (1), pág. 113-118. DOI: 10.4067/S0718-381X2015000100018.

Jímenez Órtiz, José L. Herrera Silva, Jorge C. Jiménez Órtiz, Josué. Lizárraga, Eliasib P. Murillo, José. (2017): Hallazgos incidentales en ortopantomografías maxilomandibulares de pacientes adultos jóvenes. En: Revista ADM 74 (1), pág. 25-31.

Peters, F I. et al. (2012): Prevalencia de quistes odontogénicos, Hospital Regional Valdivia entre los años 1990 y 2010. En: AVANCES EN ODONTOESTOMATOLOGÍA 28 (6), pág. 303-309.

Ponce de León Pérez, María Alejandra. (2014): Prevalencia de Hallazgos en Radiografías Panorámicas de los Pacientes de la Clínica Docente UPC Durante el Período de Febrero 2011- Abril 2014. (Tesis Cirujano Dentista). Universidad Peruana de Ciencias Aplicadas, Perú.

Sanchez Trocino, Benjamin. Hernández, Javier. Díaz Acevedo, Jacinto. Pineda, Gabriela (2013): Prevalencia de Alteraciones y Patologías en Radiografías Panorámicas en Pacientes Atendidos en una Clínica Dental Universitaria. En: Int. J. Odontostomat, 7 (1), pág. 47-52.

Sinche A, José. (2014): Hallazgos Patológicos en radiografías panorámicas previas al tratamiento de Ortodoncia en el Hospital Central FAP en el año 2012 - 2013. (Tesis Cirujano Dentista). Universidad Nacional Mayor de San Marcos, Perú.

Thiers, LC. Sotomayor, CC. Peters, Fl. et al (2013): Prevalencia de tumores odontogénicos 
en el Hospital Base Valdivia, periodo 1989-2008. En: AVANCES EN ODONTOESTOMATOLOGÍA 29 (6), pág. 303-308.

Zúñiga, Janneth. (2017): Cuantificación por Cuadrantes de la Distorsión Presente en la Radiografía Panorámica Convencional. En: Int. J. Morphol, 35 (1), pág. 265-272. DOI: 10.4067/S0717-95022017000100043. 